\title{
Lesión traqueal como hallazgo radiológico. A propósito de un caso
}

\author{
Tracheal injury as a radiological finding. Case report
}

Miguel Calva Maldonado', Samuel Margarito Lozano Camacho², Noemí Judith García Contreras ${ }^{3}$

\begin{abstract}
Tracheal injury can occur as a rare complication of endotracheal intubation, associated with multiple anatomical and mechanical factors that have been described; however, the actual incidence is unknown due to the few series of documented cases that are reported worldwide. It is considered a fatal complication when it occurs and a diagnosis is not established in a timely manner. We present the case of a patient with active SARS-CoV-2 infection and a history of congenital malformation, who presented a tracheal lesion secondary to reintubation as a radiological finding.
\end{abstract}

\section{RESUMEN}

La lesión traqueal puede ocurrir como complicación rara de una intubación endotraqueal, asociada a múltiples factores que han sido descritos de tipo anatómico y mecánico, sin embargo, la incidencia real se desconoce por las pocas series de casos documentados que se reportan a nivel mundial. Considera como una complicación mortal cuando se presenta y no se establece un diagnóstico de forma oportuna. Presentamos el caso de un paciente con infección activa de SARS-CoV-2 y antecedente de malformación congénita, que presentó como hallazgo radiológico una lesión traqueal secundaria a reintubación.

\section{Key words:}

Tracheal injury, intubation, SARS-CoV-2

\section{Palabras clave:} Lesión traqueal, intubación, SARS-COV-2

Autor, Profesor Investigador tipo C asociado BUAP, Titular de la especialidad en Anestesiología Hospital Universitario de Puebla, adscrito al Servicio de Anestesiología del Hospital General de Cholula. Puebla, México.

2 Coautor, Anestesiólogo pediatra adscrito al Hospital General de Cholula, Puebla, México; jefe de servicio de Anestesiología del Hospital para el Niño Poblano. Puebla, México.

3 Colaborador, Médico residente de cuarto año de Anestesiología Pediátrica, Hospital para el niño poblano. Puebla, México.

Fecha de ingreso: 23 de febrero de 2021

Fecha de aceptación: 03 de marzo de 2021

\section{ORCID}

0000-0002-3371-0987

\section{Correspondencia:}

Dr. Miguel Calva

drcalva55@gmail.com 


\section{Caso clínico}

S e comenta el caso de un masculino de 29 años de edad, antecedente de sindactilia izquierda, sin ningún otro antecedente patológico quien ingresó a este centro hospitalario con infección por SARS-CoV-2, con inicio de síntomas 26 días previos a su ingreso, tratado de forma privada con levofloxacino, ivermectina, ceftriaxona y dexametasona (no especificando dosis o días de tratamiento), además de oxígeno suplementario a través de puntas nasales, con deterioro clínico progresivo que requirió manejo avanzado de la vía aérea en clínica privada de referencia. Durante su estancia requiere cambio de cánula endotraqueal después de 48 h de intubación por disfunción de la misma, la cual se realiza con apoyo de guía Bougie, sin reportar ninguna complicación, manteniendo al paciente en posición prono y parámetros de ventilación elevados e insuficientes para lograr protección pulmonar; se nos interconsulta $12 \mathrm{~h}$ después, ya que el paciente presentaba nuevamente disfunción de cánula endotraqueal con saturación de $60 \%$ a pesar de $\mathrm{FiO}_{2}$ 100\%; se valora radiografía tele de tórax, donde se identifica posición disfuncional de cánula con una probable rotura y falsa vía traqueal en cara lateral izquierda (Figura 1), enfisema subcutáneo cervical y supraclavicular bilateral (Figuras 2 y 3), motivo por el cual se planea realizar cambio de cánula con apoyo de servicio de cirugía general si hubiera necesidad de realizar traqueostomía de urgencia. En laringoscopia directa con uso de dispositivo Glidescope, se identifica importante edema y friabilidad de tejidos blandos, además de edema de epiglotis, presenta dificultad para el retiro de cánula orotraqueal tipo Murphy con globo \#8.5 hasta girar $45^{\circ}$, no se identifica sangrado activo y se procede a intubación bajo visión directa con cánula orotraqueal \#7.0, verificando presencia de ventilación bilateral y elevación de niveles de saturación por arriba de 70\%, sin embargo, desarrolla arritmia que evoluciona a bradicardia menor de 30 latidos sin respuesta a adrenalina ni maniobras de reanimación cardiopulmonar avanzada, declarando fallecimiento 30 minutos después.

\section{Discusión}

La rotura traqueal puede ocurrir como complicación de una intubación endotraqueal, principalmente durante el manejo de urgencia de la vía aérea. El diagnóstico se ve retrasado como resultado de su rara incidencia en adultos y niños y la gran mayoría son casos aislados o series pequeñas con pocos pacien-

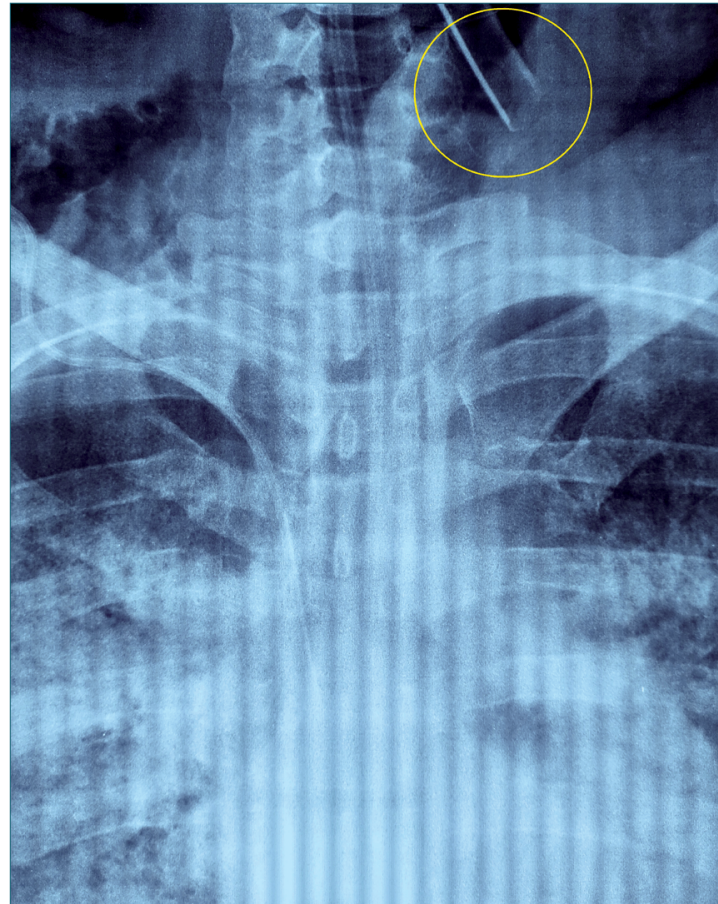

Figura 1. Falsa vía traqueal en cara lateral izquierda.

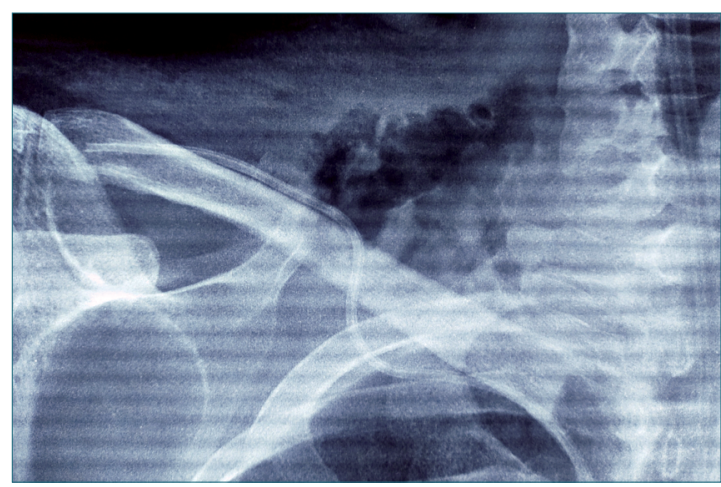

Figura 2. Enfisema subcutáneo cervical.

tes[1],[2]. La incidencia reportada en la literatura es baja $(0,05 \%-0,5 \%)[3]$, descrita en $0,005 \%$ cuando se utiliza un tubo de un solo lumen y oscila entre $0,05 \%$ y $0,19 \%$ cuando se utiliza un tubo de doble lumen. Suele producirse en el tercio distal de la tráquea y en los bronquios principales, en la unión de las porciones membranosas y cartilaginosa posterior a un traumatismo torácico, mientras que cuando tiene origen iatrogénico, suele producirse en la membrana traqueal posterior, paralelo al eje longitudinal de la tráquea; suele considerarse como una lesión potencialmente 


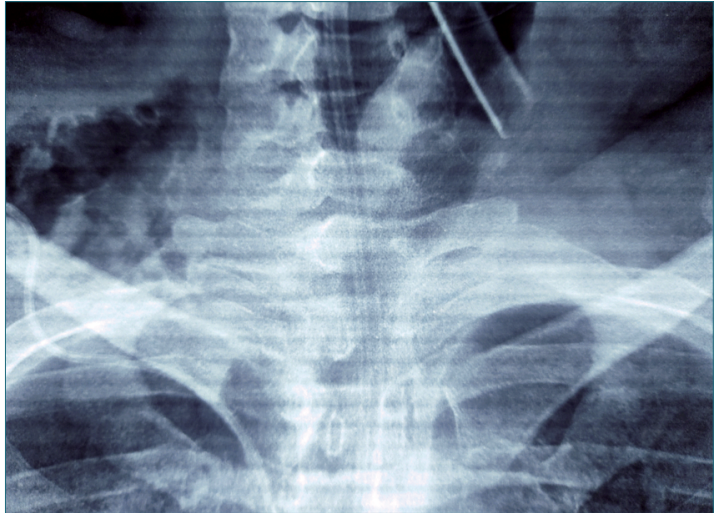

Figura 3. Enfisema supraclavicular bilateral.

mortal, cuyo diagnóstico temprano es crucial para reducir la morbimortalidad[1],[3],[4],[5].

Las lesiones traqueales pueden definirse como pérdida de solución de continuidad traqueal y de acuerdo a su extensión, puede clasificarse como ruptura traqueal (interrumpe la continuidad de la tráquea en toda su extensión), laceración traqueal (interrumpe la continuidad de la tráquea en sentido longitudinal) y lesión compleja (involucra un trayecto de dirección cambiante o lesión en alguno de los bronquios)[6].

El mecanismo exacto de la lesión es incierto y se ha propuesto un origen multifactorial, con factores de riesgo anatómicos y mecánicos[2],[7]. Los factores mecánicos incluyen: intubación de emergencia, múltiples intentos, inexperiencia, introductores de tubos endotraqueales que sobresalen más allá de la punta del tubo (se recomienda retirarlo una vez que la punta del tubo endotraqueal haya pasado las cuerdas vocales para reducir la rigidez del tubo), remoción tardía del estilete, uso de tubos de doble lumen, sobre inflación del manguito, posicionamiento incorrecto de la punta del tubo cuando la punta del mismo queda atrapada en un pliegue de la membrana traqueal posterior, reposicionamiento del tubo sin desinflar el globo, tamaño inapropiado del tubo (tubo endotraqueal más grande), tos y reposicionamiento de cabeza y cuello mientras el paciente está intubado[1],[3],[4],[8],[9],[10]. Los factores anatómicos incluyen anormalidades traqueales, debilidad de la parte membranosa de la tráquea, enfermedad pulmonar obstructiva crónica, lesiones inflamatorias traqueobronquiales, anomalías que alteran la posición de la tráquea (colecciones mediastinales, ganglios linfáticos o tumores)[2],[8], uso crónico de esteroides, edad avanzada, sobrepeso, cuello corto, sexo femenino, edad mayor a 50 años, talla menor a $160 \mathrm{~cm}$ y malformaciones congénitas[2],[3],[4],[11].

Las malformaciones congénitas de la vía aérea pueden estar asociadas a sindactilia en los siguientes casos: síndrome de Fraser (estenosis laríngea), síndrome de Pfeiffer (estenosis traqueal), síndrome de Apert - acrocéfalo-sindactilia (asociada a la presencia de fístula traqueoesofágica así como alteraciones en la flexibilidad de la columna cervical por fusión de C5C6, haciendo de la intubación orotraqueal un procedimiento complicado), síndrome de Larsen (traqueomalacia y estenosis subglótica), síndrome de Pallister Hall (epiglotis bífida, hipoplasia de epiglotis y displasia de cartílagos traqueales)[12],[13],[14],[15],[16].

Las manifestaciones clínicas más comunes en general son enfisema subcutáneo en tórax y cuello, enfisema mediastinal (si la lesión es intratorácica, en cuyo caso puede estar presente el signo de Hamman, descrito como la presencia de crépito precordial concordante con cada latido cardíaco, que se ausculta con el paciente en decúbito lateral), neumotórax e insuficiencia respiratoria, así como disnea tras la extubación[4],[17],[6]. El diagnóstico debe sospecharse cuando se desarrolla enfisema subcutáneo en tejidos blandos de cara, cuello, hombros o tórax[1]. Las primeras manifestaciones pueden aparecer de 2 hasta 120 h después de que la lesión ocurre[6]. El enfisema subcutáneo masivo puede poner en riesgo la vida, al generar obstrucción del flujo de aire, disminución del retorno cardíaco, mala perfusión a cabeza y cuello, rigidez de la pared torácica secundaria por disminución en la expansión pulmonar[11],[18]. Otros signos incluyen enfisema palpebral, disfonía, disnea, disfonía, tos, hemoptisis, aumento agudo de la circunferencia de cuello, neumoperitoneo que se desarrollan inmediatamente o poco después de la extubación, aunque pueden tardar varios días en aparecer[2],[6]. $\mathrm{Si}$ el diagnóstico se retrasa, el paciente puede desarrollar mediastinitis y sepsis secundaria a lesión traqueal[11],[18]. El diagnóstico se confirma mediante broncoscopia flexible, lo que permite establecer una ubicación, extensión y profundidad precisa[8]. La radiografía de tórax y cuello puede aportar información para reforzar la sospecha diagnóstica encontrando muchas veces presencia de un tubo endotraqueal sobredistendido, con orientación oblicua y a la derecha distal de la carina, enfisema subcutáneo, mediastinal y neumotórax[6]. La tomografía puede revelar una lesión traqueal y ser útil para tomar decisiones sobre el tratamiento[1].

En estudios de Ross cols. y Massard cols, las indicaciones de tratamiento conservador se reservan para enfisema mediastinal no progresivo y/o enfisema subcutáneo, con lesión menor de $5 \mathrm{~cm}$ de longitud, 
sin comunicación importante con el espacio mediastinal, ausencia de lesiones esofágicas, signos vitales estables y contraindicación absoluta por trastornos concomitantes[1],[3],[4]. En pacientes que requieren ventilación mecánica, esta cicatrización puede verse afectada por la presencia del manguito descansando sobre la lesión y la presión positiva ejercida sobre la vía respiratoria[19].

La corrección quirúrgica se indica en el primer signo de inestabilidad respiratoria o si hay evidencia de mediastinitis, así como en pacientes con lesiones mayores a $2 \mathrm{~cm}$, que requieren ventilación mecánica, o que presentan neumotórax y enfisema subcutáneo progresivo, lesiones esofágicas o comunicación importante con el espacio mediastinal, si la lesión es proximal y pequeña, el tratamiento quirúrgico no resulta complejo; en cambio, si la lesión afecta a uno de los bronquios, requiere realización de traqueostomía que permita una intubación selectiva y repara- ción quirúrgica de la lesión[1],[3],[4],[19].

\section{Conclusiones}

El conocer los factores de riesgo asociados a la presentación de lesión traqueal, permitirá al anestesiólogo prevenir la aparición de esta complicación rara y potencialmente devastadora, así como identificar los signos clínicos y radiológicos de sospecha para establecer un tratamiento de forma oportuna. Sin embargo, en el contexto de la pandemia actual por SARS-CoV-2, se tienen limitantes, que asociadas a las características clínicas de estos pacientes, condicionan un retraso en el diagnóstico que demora el establecer un tratamiento expedito y adecuado. Es por eso que se da a conocer este caso clínico, esperando sea de utilidad como referencia en caso de presentar un caso similar en la práctica médica diaria.

\section{Referencias}

1. Lung Chang Yin et.al. Case report. Tracheal Rupture After Emergent Endotracheal Intubation. Tzu Chi Medical Journal. September 2008 Vol 20 No. 3

2. Miñambres E, Burón J, Ballesteros MA, Llorca J, Muñoz P, González-Castro A. Tracheal rupture after endotracheal intubation: a literature systematic review. Eur J Cardiothorac Surg. 2009 Jun;35(6):1056-62. https://doi. org/10.1016/j.ejcts.2009.01.053 PMID:19369087

3. Mantovani S, Poggi C, Bassi M, Cagnetti S, Anile M, Venuta F. Surgical or conservative management of post intubation tracheal injury: when and how? J Vis Surg. 2020;6:4. https://doi. org/10.21037/jovs.2019.09.07.

4. Medina CR, Camargo JJ, Felicetti JC, Machuca TN, Gomes BM, Melo IA. Post-intubation tracheal injury: report of three cases and literature review. J Bras Pneumol. 2009 Aug;35(8):80913. https://doi.org/10.1590/
S1806-37132009000800014 PMID:19750335

5. Juneja P, Ghiassi K, Ramaswamy S, Kanoff J. latrogenic tracheal rupture post endotracheal intubation causing pneumomediastinum in a Down Syndrome patient. Chest. 2020 Oct;158(4 4s):A1920. https://doi.org/10.1016/j. chest.2020.08.1663.

6. Angeles B, Carlos J. Lesiones traqueales iatrogénicas relacionadas con el abordaje de la vía respiratoria. Revista Mexicana de Anestesiología. 2018 OctDec;4(4):273-7.

7. Xu X, Xing N, Chang Y, Du Y, Li Z, Wang Z, et al. Tracheal rupture related to endotracheal intubation after thyroid surgery: a case report and systematic review. Int Wound J. 2016 Apr;13(2):268-71. https:// doi.org/10.1111/iwj.12291 PMID:24871935

8. Aguilar R. Singh. The radiological features of tracheal rupture following endotracheal intubation. Imaging Med. 2017;9(5).
9. Evans D, McGlashan J, Norris A. latrogenic airway injury. BJA Educ. 2015;15(4):184-9. https:// doi.org/10.1093/bjaceaccp/ mku026.

10. Chang CY, Cheng SL, Chang SC. Conservative treatment of severe tracheal laceration after endotracheal intubation. Respir Care. 2011 Jun;56(6):861-2. https:// doi.org/10.4187/respcare.00891 PMID:21333080

11. Miñambres E, Burón J, GonzálezCastro A, Rodríguez-Borregán JC, Mons R, López-Espadas F. Rotura traqueal tras intubación urgente. Med Intensiva. 2005;29(7):393-5. https:// doi.org/10.1016/S02105691(05)74268-6.

12. Sierra B, Melissa C, et al. Síndrome de Fraser: reporte de un caso. Rev Mex Pediatr. 2019;86(4):155-8.

13. Arce R, Jorge y Villarroel C. Camilo. Síndrome de Pfeiffer tipo 2. Informe de un caso y revisión de la literatura. Acta Pediatr Mex. 2013;34(1):43-7.

14. Goytia V. Alfredo et al. Síndrome 
de Apert: caso clínico (acrocefalosindactilia). Gac Med Bol. Vol 30. No.1, 2007. Pp 58-62.

15. Aparicio J. Irene. Implicaciones anestésicas en el síndrome de Larsen: A propósito de un caso. Revista electrónica de Anestesiar. Vol 11, No. 10; 2019.

16. Chapter 4. In: Biesecker G. Leslie. Cassidy and Allanson's Management of Genetic Syndromes; 2020.
17. Keenan A, Browne W, Bhargava M. Diffuse Subcutaneous Emphysema after tracheal perforation. Am J Respir Crit Care Med. 2017 May;195(10):1397-8. https:// doi.org/10.1164/rccm.2016112394IM PMID:28306325

18. Gries CJ, Pierson DJ. Tracheal rupture resulting in life-threatening subcutaneous emphysema. Respir Care. 2007 Feb;52(2):191-5.

\section{PMID:17261208}

19. Wallet $F$, Schoeffler $M$, Duperret S, Robert MO, Workineh S, Viale JP. Management of low tracheal rupture in patients requiring mechanical ventilation for acute respiratory distress syndrome. Anesthesiology. 2008 Jan;108(1):159-62. https://doi.org/10.1097/01. anes.0000296104.46682.ca PMID:18156895 